\title{
Stability, spatial extension and extinction of an electric arc in aeronautical conditions of pressure under $540 \mathrm{~V}$ DC
}

\author{
Romaric Landfried ${ }^{1, *}$, Mohamed Boukhlifa $^{1}$, Thierry Leblanc ${ }^{1}$, ${\text { Philippe } \text { Teste }^{1} \text {, and Jonathan Andrea }}^{2}$ \\ ${ }^{1}$ GeePs | Group of Electrical Engineering - Paris, CNRS, CentraleSupélec, Univ. Paris-Sud, Université Paris-Saclay, \\ Sorbonne Université, 3 \& 11 rue Joliot-Curie, Plateau de Moulon, 91192 Gif-sur-Yvette Cedex, France \\ ${ }^{2}$ Leach Esterline Power System, Esterline Power Systems Secondary Electrical Distribution Department, Sarralbe, France
}

Received: 11 February 2019 / Received in final form: 26 June 2019 / Accepted: 9 September 2019

\begin{abstract}
This work deals with the characterization of DC electric arcs in aeronautical conditions of pressure (from $10^{4} \mathrm{~Pa}$ to $10^{5} \mathrm{~Pa}$ ). Observations have been made with the help of a high speed camera and various characteristics of electric arc under $540 \mathrm{~V}$ DC have been studied: the stability arc length, the extinction gap, the arc duration and the mean energy dissipated in the arc. The arc current intensity range is 10-100 A. The obtained results show that the arc stability length, extinction electrode gap, arc duration and energy dissipation in the arc have a direct correlation with the pressure and the current in the circuit.
\end{abstract}

\section{Introduction}

The entire aviation industry is mobilized to the ambition of More Electric Aircraft (MEA) concept, in which the three secondary energies will be replaced by the only electrical energy. Currently, most embedded networks are powered at $110 \mathrm{~V} \mathrm{AC}$ and $28 \mathrm{~V} \mathrm{DC}$. The voltage level will increase to $230 \mathrm{~V} \mathrm{AC}$ and $\pm 270 \mathrm{~V}$ DC or $0-540 \mathrm{~V}$ DC. This evolution will have many consequences in various areas. Among the inescapable consequences, some will touch the field of electric switching. Indeed, the future switching-off devices will have to operate under continuous voltages of about $270 / 540 \mathrm{~V}$ DC, but will have also to work under low pressure conditions (from $2 \times 10^{4}$ to $10^{5} \mathrm{~Pa}$ ). In $\mathrm{AC}$ regime the arc will extinguish either when contacts moves away increasing the arc voltage and then the voltage needed to sustain this one or when the current flows to zero (every half period) which provides an additional opportunity to extinguish the arc. However, in DC regime the arc extinction occurs only when the required voltage to sustain the arc becomes greater than what the supply circuit may produce. Under $28 \mathrm{~V} \mathrm{DC}$, the natural arc extinction occurs for an arc length near a few millimeters. For instance, in the case of $36 \mathrm{~V}$ DC supply voltage, it has been observed [1] that an order of magnitude of the spatial extension of an electric arc was about $6 \mathrm{~mm}$ at atmospheric pressure and $10 \mathrm{~mm}$ at $2 \times 10^{4} \mathrm{~Pa}$.

Another consequence of the increase of the aircraft network voltage concerns the field of electric arc faults. Faults in power grids can generate electric arcs that can reach high current intensities. Their durations and their spatial extensions are limited either by protective devices

\footnotetext{
* e-mail: romaric.landfried@supelec.fr
}

or by natural extinction of the arc. However, the service life of an arc fault and consequently the material damage caused in its immediate environment can be significant. They can lead to a loss of functionality, damage the system, spread and even cause a fire in a few seconds (more than $60 \%$ risk of fire starting for an arc that lasts $4 \mathrm{~s}$ under 120 $240 \mathrm{~V}$ AC). This problem, common to many power grids when supply voltages exceed some tenths volts, takes a critical aspect in terms of consequences in the field of avionics. Their causes of occurrence are multiple. These can be, cut cables, mechanical problems such as an unexpected contact between two conducting parts, occurrence of partial discharges which are able to transit spontaneously to electric arcs, leakage current in a printed circuit which can lead over time to the progressive appearance of a major heating, and induce an electric arc. To guard against the direct effects of such defects (such loss of functionality) in the aeronautical field, many precautions are taken (redundancies circuits and wiring, etc.). As for the indirect effects (damage, risk of fire), they are limited by the use of circuit breakers. Despite the large number and effectiveness of these protection systems, arcs can occur. Two interrelated phenomena are particularly important for the damages created by the arc: the spatial extension that the arc can take and its propagation, for example in PCB, in a bundle of cables.

In the case of low voltage value $(28 \mathrm{~V} \mathrm{DC})$, the electric arc interacts only with its very near environment and the risk of damaging nearby components (cables, connector, hull, etc.) remains reduced. In the case of much higher supply voltage values it is necessary to observe the behavior of possible arc fault (spatial extension, stability of the arc). The voltage level increase to $\pm 270 \mathrm{~V}$ DC or 0-540 V DC will strongly enhance the possible damages caused by arc faults. For instance, the likelihood of a fire 
ignition due to an arc fault, in presence of fuel spray has been studied either for $28 \mathrm{~V} \mathrm{DC}$ or $270 \mathrm{~V}$ DC power supply voltage at atmospheric pressure. As well as in the case of series arcs or parallel arcs, it was observed that the increase of the supply voltage from $28 \mathrm{~V} \mathrm{DC}$ to $270 \mathrm{~V}$ DC causes a major increase of the number of fire ignition cases [2].

Under these voltage conditions, not only the initiation of an arc will be easier, but maintaining it will be facilitated, its spatial extension will greatly be increased and concomitantly the ability of the arc to spread will also be strongly impacted. The results presented in this paper have been obtained for a pressure varying in the range $10^{4}-10^{5} \mathrm{~Pa}$. It may be recalled that a pressure of $2 \times 10^{4} \mathrm{~Pa}$ corresponds commercial airplane cruise altitude $\left(12000 \mathrm{~m}\right.$ ), and $10^{4} \mathrm{~Pa}$ (equivalent to $15000 \mathrm{~m}$ ) is often considered for certification. Results for this pressure could then be interesting. Studying and characterizing arc phenomena in this pressure range is then an important challenge to accompany this change in embedded networks, to bring information concerning arc switching off properties, to better understand the risks involved and to improve the means of remedying them in terms of detection and protection.

The purpose of the paper proposed here is to present a first study concerning the properties of an electric arc under 540 V DC supply voltage in sub-atmospheric pressure in the range $\left[10^{4}-10^{5}\right] \mathrm{Pa}$.

The paper is organized as follows:

- A first part will be dedicated to the bibliography concerning properties of DC electric arcs. Most of these previous works were done at atmospheric pressure for the automotive field. The voltages implemented are of the order of 36-42 V DC.

- In the second part, the experimental device implemented is described.

- In the third part experimental results are presented. They concern the influence of various parameters on the spatial extension, the extinction and the energy dissipated in such an electric arc. These parameters taken into account are the following: the supply voltage, the intensity of the arc current, the pressure value, etc.

\section{Rapid review concerning some properties of electric arc under DC voltage}

Works on arcs in DC mode under voltages of a few tens to a few hundred volts in aeronautical conditions of pressure are not numerous. For high supply voltages $(540 \mathrm{~V}$ DC for example), it is necessary to go back to the 1950s [3-6] to find works dealing with our experimental conditions. These works concerned traction systems and avionics. In this field it was a question of characterizing components (contactors, circuit-breakers), and the possible over voltages occurring during breakdown in the presence of a high inductance. More recently some works have been studying the influence of pressure on usual switching devices [7]. However, most of recent works concerning electric arc in DC regime are related to the evolution of embedded voltages $(36-42 \mathrm{~V}$ DC) in the automotive field. The simplest way to study the spatial extension of an electric arc is to drive experiments by increasing the distance between the electrodes and

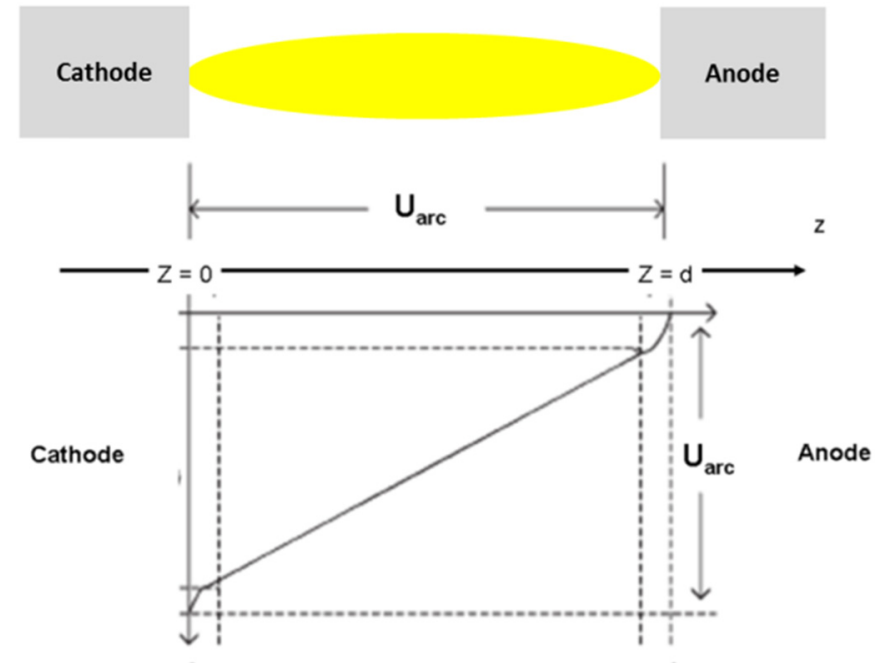

Fig. 1. Schematic representation of an ideal electric arc and voltage distribution in the arc column.

observing the distance at which the extinction of the arc occurs. This distance will depend on various parameters such as the separation dynamics of the electrodes, the nature and the pressure of the plasma gas as well as the load in the circuit (presence or absence of inductance). Several authors have proposed expressions giving the maximum length of the arc according to various parameters such as the supply voltage, the opening speed and the intensity of the arc current. Most of these works were done at atmospheric pressure for the automotive field. The conducted studies concern low-intensity $\operatorname{arcs}(I<10 \mathrm{~A})$ at voltages in the range of $14-42 \mathrm{~V} \mathrm{DC} \mathrm{[8].} \mathrm{Most} \mathrm{of} \mathrm{the} \mathrm{time}$ the electrode material was used in the electrical contact field: $\mathrm{Au}, \mathrm{Ag}$ or $\mathrm{Ag}-\mathrm{MeO}$ type $\left(\mathrm{Ag}-\mathrm{SnO}_{2}\right.$ or $\mathrm{Ag}-\mathrm{CdO}$ for instance) $[9,10]$. In these experimental conditions, the maximum length of the arc may reach some millimeters and it is an increasing function of the arc current intensity. Rare works concern copper electrodes and higher supply voltage $[11,12]$. In this case $\left(I_{\text {arc }}>100 \mathrm{~A}\right)$ it was observed that the length of the arc was not necessarily an increasing function of the intensity of the current, but that it appeared for high intensities, electromagnetic instabilities which deform the arc column. More recently, academic works $[1,13]$ have been carried out to characterize electric arcs for pressure in the range $\left[10^{4}-10^{5}\right] \mathrm{Pa}$ for voltages of the order of 36-42 V DC and arc current of the order of a few tens of amperes.

In the case of an "ideal" electric arc (axial symmetry) the arc voltage versus the arc column length is plotted and takes the following well known expression:

$$
U_{\text {arc }}=V_{A}+V_{C}+\int_{0}^{d}|E(z)| d z
$$

where $U_{\text {arc }}$ is the arc voltage, $V_{A}$ and $V_{C}$ the anode and cathode voltage fall respectively, $d$ the arc length and $E$ the electric field in the arc column.

In Figure 1, a schematic representation of an ideal electric arc is given with the axial voltage distribution in the arc column. 


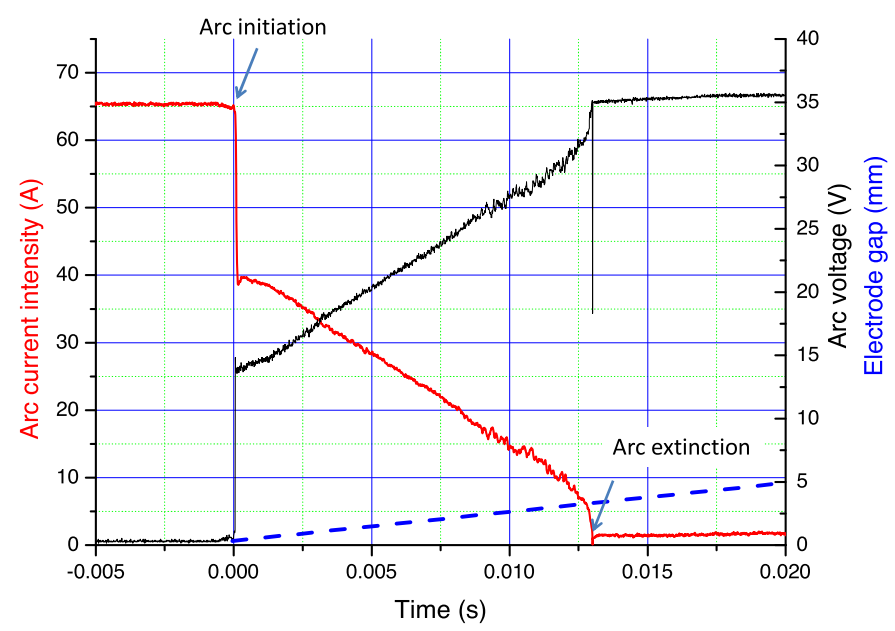

Fig. 2. Arc current intensity and arc voltage evolutions in the case of an electric arc burning between 2 copper electrodes in air at atmospheric pressure for a supply voltage equal to $36 \mathrm{~V} \mathrm{DC}$.

Table 1. Mean electric field in the arc column - results extracted from [1].

\begin{tabular}{lll}
\hline & $I_{\text {arc }} \approx 30 \mathrm{~A}$ & $I_{\text {arc }} \approx 100 \mathrm{~A}$ \\
\hline$P=250 \mathrm{mbar}$ & $2.3-4.7 \mathrm{kV} / \mathrm{m}$ & $1.4-1.9 \mathrm{kV} / \mathrm{m}$ \\
$P=1 \mathrm{bar}$ & $4.7-5 \mathrm{kV} / \mathrm{m}$ & $1-2.1 \mathrm{kV} / \mathrm{m}$ \\
\hline
\end{tabular}

In DC regime, the electric arc extinguishes when the voltage required to maintain the electric arc is greater than the voltage that the circuit (power supply and load) may produce. Considering a non-inductive circuit, the maximal value of the arc length, denoted $d_{\max }$, is schematically given by:

$$
d_{\max }=\frac{U_{\text {sup }}-\left(V_{A}+V_{C}\right)}{\left|E_{\text {col }}\right|}
$$

where $U_{\text {sup }}$ is the supply power voltage and $E_{\text {col }}$ the mean electric field in the arc column.

The record of arc current intensity and voltage evolutions under a supply voltage of $36 / 42 \mathrm{~V}$ DC is presented in Figure 2. At the arc initiation due to the electrode separation, the current intensity strongly decreases $(-35 \%)$ and continues to regularly decrease during the separation. The arc duration remains quite short and the switch off (corresponding to the length of extinction) occurs for an electrode gap approximatively equal to $3 \mathrm{~mm}$. Under this value of supply voltage, only very small arcs may occur because the supply voltage value is of the same order of the sum of electrode voltage fall noted $\left(V_{A}+V_{C}\right)$. In the case of small arcs, the arc has little instability. This is noticeable in the shape of the voltage which is smooth. The arc model proposed in Figure 1 then applies (some millimeters in length for a $36 \mathrm{~V}$ DC supply voltage). $E_{\mathrm{col}}$ values have been measured [1] in aeronautical conditions of pressure. Results are briefly summarized in Table 1.

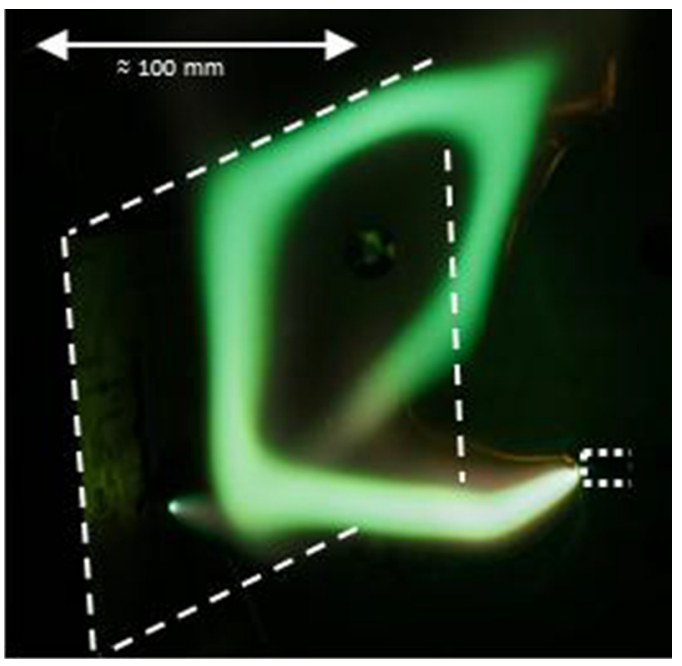

Fig. 3. Photography of an electric arc burning in air $P=2 \times 10^{4} \mathrm{~Pa}$ and $I_{\mathrm{CC}} \approx 100 \mathrm{~A}$ under $540 \mathrm{~V} \mathrm{DC}$.

Using these values to extrapolate the arc length for $U_{\text {sup }}=540 \mathrm{VDC}$ generates an important dispersion in $d_{\max }$ value. For instance for $I_{\text {arc }} \approx 30 \mathrm{~A}$ and $P=2.5 \times 10^{4} \mathrm{~Pa} d_{\max }$ will be in the range [11-22] $\mathrm{cm}$ and for $I_{\text {arc }} \approx 100 \mathrm{~A}$ an estimation of $d_{\max }$ is in the range [28-38] $\mathrm{cm}$.

Moreover, for long electric arc column, instabilities appear and the arc may be very chaotic as it is illustrated in Figure 3 which is a photography of an electric arc between copper squared plate (on the left with $20 \mathrm{~cm}$ for each side) and a copper cylindrical electrode (on the right with a $8 \mathrm{~mm}$ diameter) for $P=2 \times 10^{4} \mathrm{~Pa}$ and $I_{\mathrm{CC}} \approx 100 \mathrm{~A}$ (before arc). Not only does the arc not have an axisymmetric shape, but also the radial extension of the arc can be very important (in the order of the inter-electrode distance).

The aim of this work is then to propose measurement of such characteristics in the case of $540 \mathrm{~V}$ DC supply.

\section{Experimental device}

The objective of the device implemented is to allow the electrical characterization of an electric arc under controlled pressure conditions. The arc can be initiated in different ways: loss of electrical continuity in a circuit (broken cable, connection or faulty connector) which generates a series arc, progressive appearance of a conductive passage (carbon bridge, salt bridge, etc.) which then generates a parallel arc or overvoltage apparition in the circuit. However, whatever the cause or mode of arc initiation, the spatial extension of the arc will mainly depend on parameters such as the supply voltage, the pressure, the arc current intensity. Thus, we have opted with a method that allows to initiate a series arc by causing a break in the electrical continuity of a circuit. This could for example correspond in terms of arc faults to the connection failure or the breaking of a cable. The electrode material chosen is OFHC (Oxygen Free High Conductivity) copper.

The device can thus be divided into two main parts:

- An electrical part schematically presented in Figure 4. - A mechanical part for which a photography is proposed in Figure 5. 


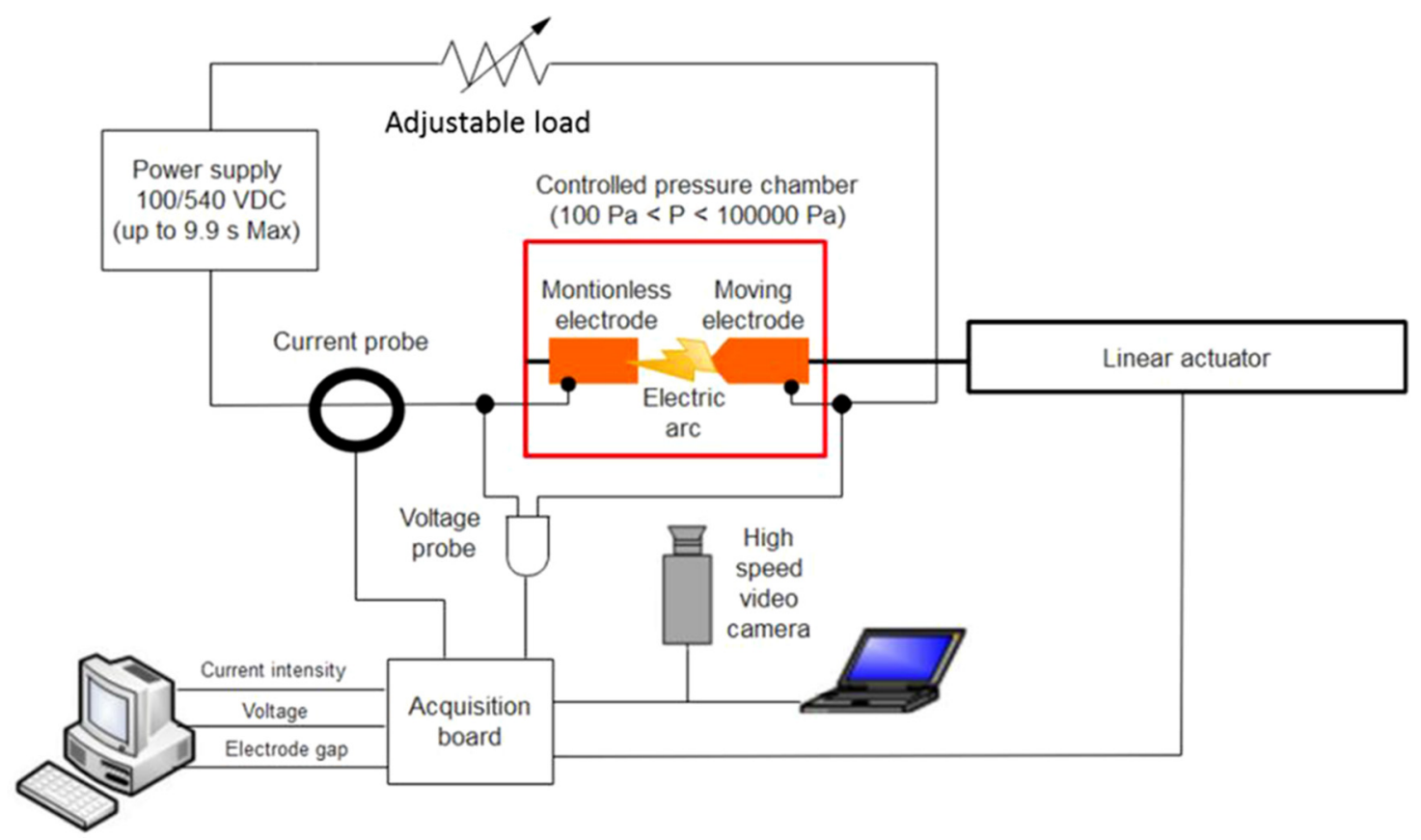

Fig. 4. Schematic electrical diagram of the experimental system.

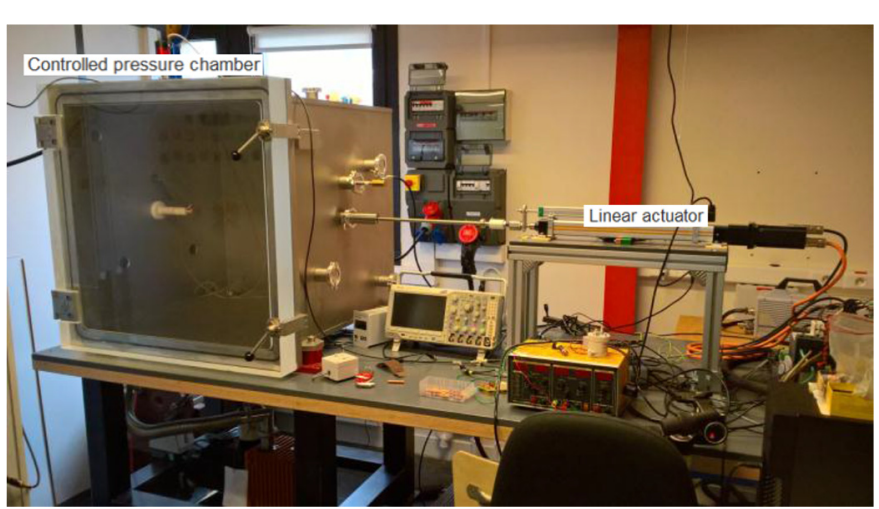

Fig. 5. Photography of the mechanical part of the experiment.

\subsection{Electrical device}

The pulse generator is based on a rectifier system (AC/DC) connected to 6 supercapacitors $(12 \mathrm{~V}$ DC) which are connected to a DC/DC convertor designed to have an output voltage between 100 and $540 \mathrm{~V}$ DC. It can provide a current intensity up to $300 \mathrm{~A}$ on a closed circuit (denoted $\left.I_{\mathrm{CC}}\right)$. In this study the electric load used is a non-inductive adjustable resistance. The load allows to adjust current intensities as we wish. An electronic device allows the adjustment of the duration of the current pulse (up to $9.9 \mathrm{~s}$ ) and thus ensures redundant security in the event that the arc does not switch off "naturally" during the separation of the contacts.

\subsection{Mechanical device}

The mechanical device consists of two coaxial OFHC copper cylindrical electrodes. The cathode, a $40 \mathrm{~mm}$ diameter cylindrical electrode, remains fixed (on the left side of each photograph) and the anode, a $8 \mathrm{~mm}$ diameter with a conical tip (the curvature radius is around $5 \mathrm{~mm}$ ), is movable. It is important to note that electrodes have been reshaped every time their shape has been modified due to erosion. This reshape is done with abrasive paper and electrodes are cleaned with $95 \%$ ethanol. A controlled electric screw-jack allows opening at adjustable speed (up to $0.6 \mathrm{~m} / \mathrm{s}$ ). The maximum opening distance is approximately $0.35 \mathrm{~m}$. The assembly is installed in a vacuum chamber $(0.75 \mathrm{~m} \times 0.75 \mathrm{~m} \times 0.75 \mathrm{~m})$ equipped with a pumping system making it easy to reach the pressures of the aeronautical field (from $10^{3}$ to $10^{5} \mathrm{~Pa}$ ). The door of the polycarbonate enclosure allows observation of the arc structure.

It is clear that such a protocol allows the study of the conditions of arc extinction. With regard to arcing defects, this protocol does not describe arcing defects that may occur in cases such as cable injuries, but it does allow the study of defects related to untimely disconnections and also allows the study of the spatial extension that arcing defects can take.

\subsection{Measurements and observations}

The following data are automatically recorded:

- the current intensity is measured across the load resistance using a differential probe (bandwidth of $70 \mathrm{MHz}$ ),

- the arc voltage is measured using a similar differential probe,

- the electrode gap is measured using a potentiometer which allows an accuracy on the gap measurement of $\pm 100 \mu \mathrm{m}$. In a previous article [1], the accuracy of the inter-electrode distance measurement was better $( \pm 13 \mu \mathrm{m})$ but the maximum measurable distance was much smaller than that reached during this work. 


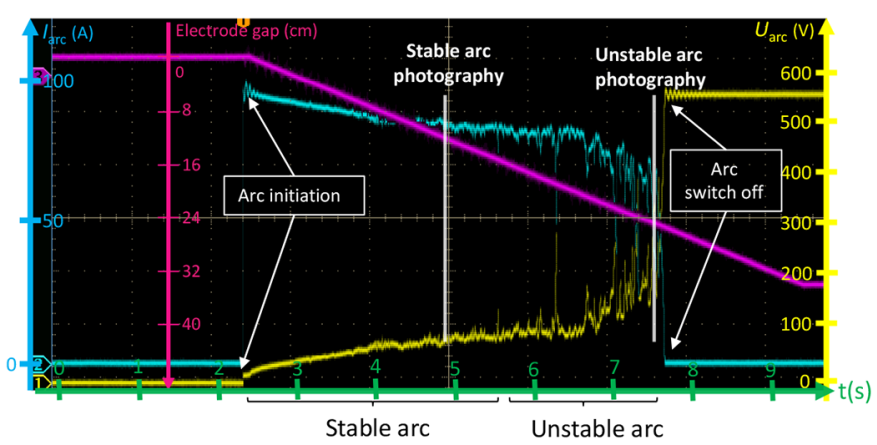

Fig. 6. Arc current intensity (in blue), arc voltage (in yellow) and electrode gap (in purple) evolutions in the case of electric arc burning between 2 copper electrodes in air $\left(I_{\mathrm{CC}} \approx 100 \mathrm{~A}\right.$, $\left.P=2 \times 10^{4} \mathrm{~Pa}\right)$. The power supply voltage is $540 \mathrm{~V} \mathrm{DC}$.

Several data are determined a posteriori:

- the maximal electrode gap. The arc ignition is detected by a strong increase in the voltage value and the extinction is detected when the current intensity reaches zero. This value does not correspond to the maximal length of the arc column because the arc may have very perturbed shapes (cf. Fig. 3).

- the energy dissipated in the arc,

- the "stability length of the arc" which has been defined as the electrode gap for which first voltage instabilities occur. It is obvious that this value cannot be defined with precision. However, beyond this distance, the arc can extinguish by elongation of its column.

To determine the maximal electrode gap and the energy dissipated in the arc, it is necessary to determine the exact ignition instant and the extinction instant. The arc ignition is detected by a strong increase in the voltage value and the extinction is detected when the current intensity reaches zero.

\section{Results}

As previously presented, the arc voltage, the current intensity and the electrode gap have been measured for several experimental conditions. Three measurements have been done for each set of every parameter.

\subsection{Observation of the electric arcs and examples of records}

In Figure 6 a typical example of the evolutions of the current intensity (blue curve), of the arc voltage (yellow curve) is given for a current intensity before the opening phase (denoted $I_{\mathrm{CC}}$ ) equal to $100 \mathrm{~A}$ and a pressure equal to $P=2 \times 10^{4} \mathrm{~Pa}$.

Unlike what happens at $36 \mathrm{~V}$ DC (see Fig. 2), the arc current intensity varies little when opening. It also decreases quite slowly during opening $(-20 \%$ for an opening of about $20 \mathrm{~cm}$ ).

Two phases may be distinguished:

- a first time interval during which the voltage signal increases "smoothly" in relation to the electrode gap increase. In this phase the electric arc is stable as it may

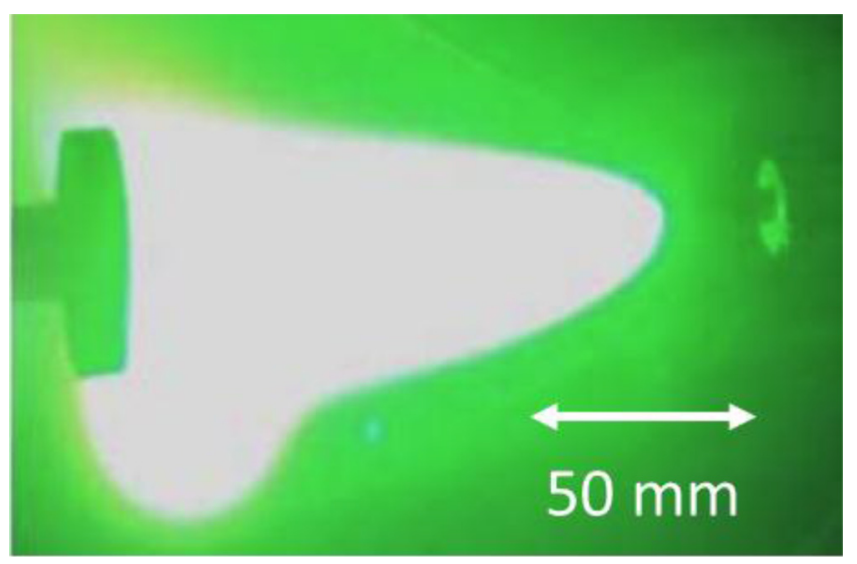

Fig. 7. Photography of a stable electric arc $\left(I_{\mathrm{CC}} \approx 100 \mathrm{~A}\right.$, $\left.P=2 \times 10^{4} \mathrm{~Pa}\right)$.

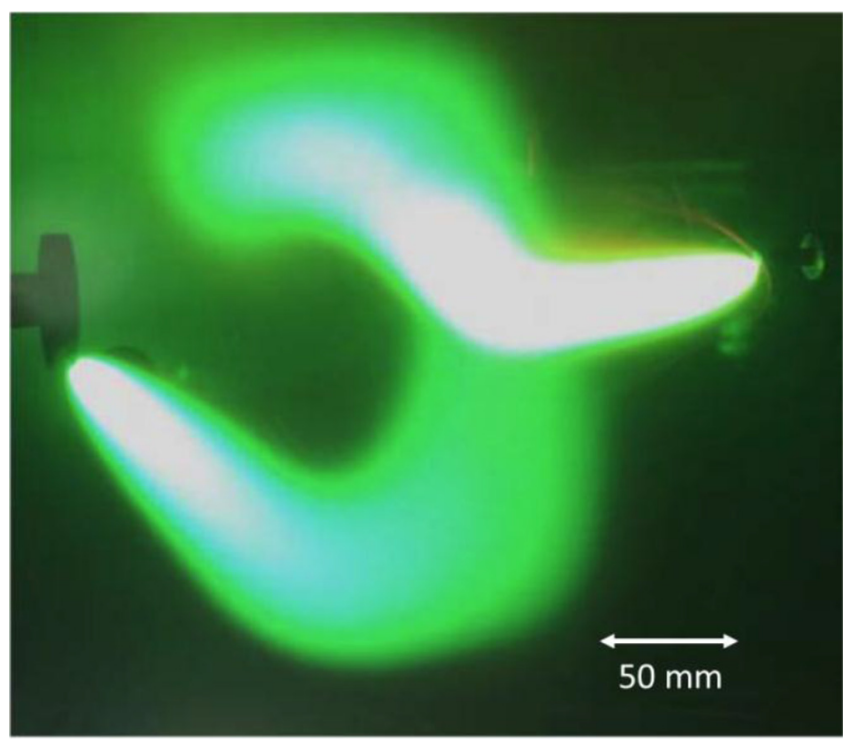

Fig. 8. Photography of an unstable electric arc $\left(I_{\mathrm{CC}} \approx 100 \mathrm{~A}\right.$, $\left.P=2 \times 10^{4} \mathrm{~Pa}\right)$.

be observed in Figure 7 which is a photography of the electric arc during this phase. The photography instant has been marked with the help of a white line in Figure 6. Figure 7 shows an electric arc which has a quasi-axisymmetric shape, which is called "stable" in this work. Its length is about $125 \mathrm{~mm}$ and its transverse extension is in the range [55-110] $\mathrm{mm}$.

- In a second phase instabilities appear. These instabilities result concomitantly in a significant fluctuation of the arc voltage (40 volts or more) and a sudden elongation of the arc column. In Figure 8, a photography of an "unstable" arc is given (also marked with the help of a white line in Fig. 3). The electrode gap is equal to $250 \mathrm{~mm}$ but the arc length is much greater. The transverse extension is of the same order of magnitude. 


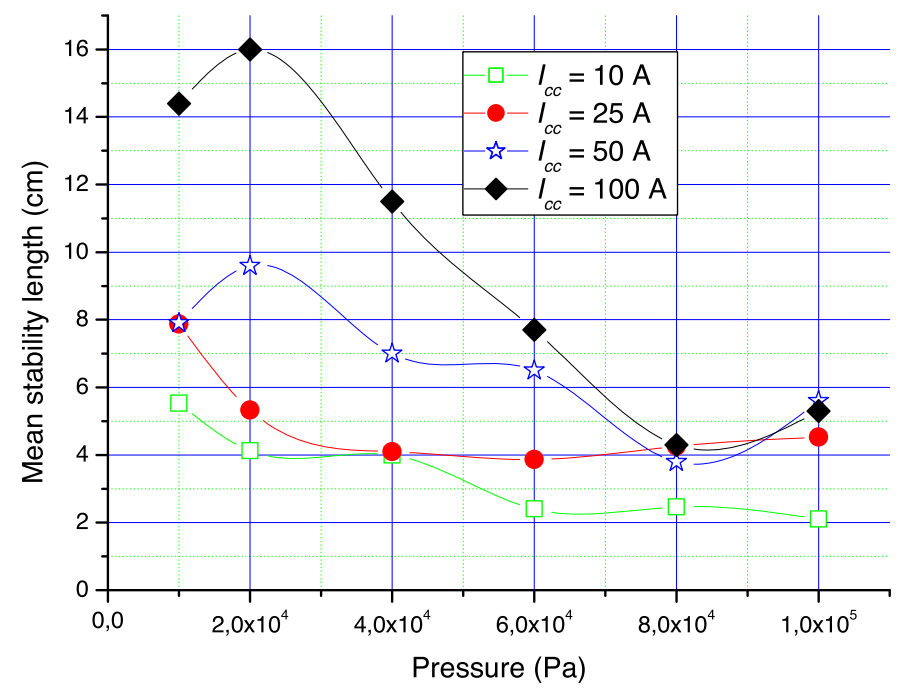

Fig. 9. Mean value of the stability length of the arc versus the pressure value, for various $I_{\mathrm{CC}}$ values: $10,25,50$ and $100 \mathrm{~A}$. The opening velocity is $50 \mathrm{~mm} / \mathrm{s}$.

\subsection{Estimation of the stability length of the arc}

In this part, we present results concerning the stability length of the arc (defined in Sect. 3.3). We have plotted in Figure 9 the mean "stability length of the arc" versus the pressure for various $I_{\mathrm{CC}}$ values: $10,25,50$ and $100 \mathrm{~A}$. In this figure, only the minimum value for the mean stability length has been plotted so that at one point corresponds one measure. Below this stability length value, the arc is stable and it can be considered that in most cases the length of the arc column is almost equal to the distance between electrodes. In this case, the separation velocity was equal to $50 \mathrm{~mm} / \mathrm{s}$.

Overall the stability of the arc is all the more important as the pressure is low. Moreover, the arc stability appears as an increasing function of the current intensity.

For every current values considered in this work, electric arc is even more unstable as the pressure is high and the mean stability length is in the range $[2-16] \mathrm{cm}$. The influence of arc current intensity is more pronounced for pressure values below $6 \times 10^{4} \mathrm{~Pa}$.

It is interesting to note that under this pressure value, the arc is all the more stable as the current intensity is high.

For an opening velocity equal to $600 \mathrm{~mm} / \mathrm{s}$, no instability in the arc voltage was observed before extinction occurs (the stability length is then substantially equal to the inter-electrode distance for which extinction occurs). We have then not drawn it.

\subsection{Extinction electrode gap and duration versus pressure}

In Figures 10 and 11, the electrode gap for which the arc extinction occurs has been plotted versus pressure for various $I_{\mathrm{CC}}$ values $(10,25,50,100 \mathrm{~A})$ and two separation velocities of the electrodes: 50 and $600 \mathrm{~mm} / \mathrm{s}$. Moreover, the electrode gap when extinction occurs has been plotted (top right in both figures) versus $I_{\mathrm{CC}}$.

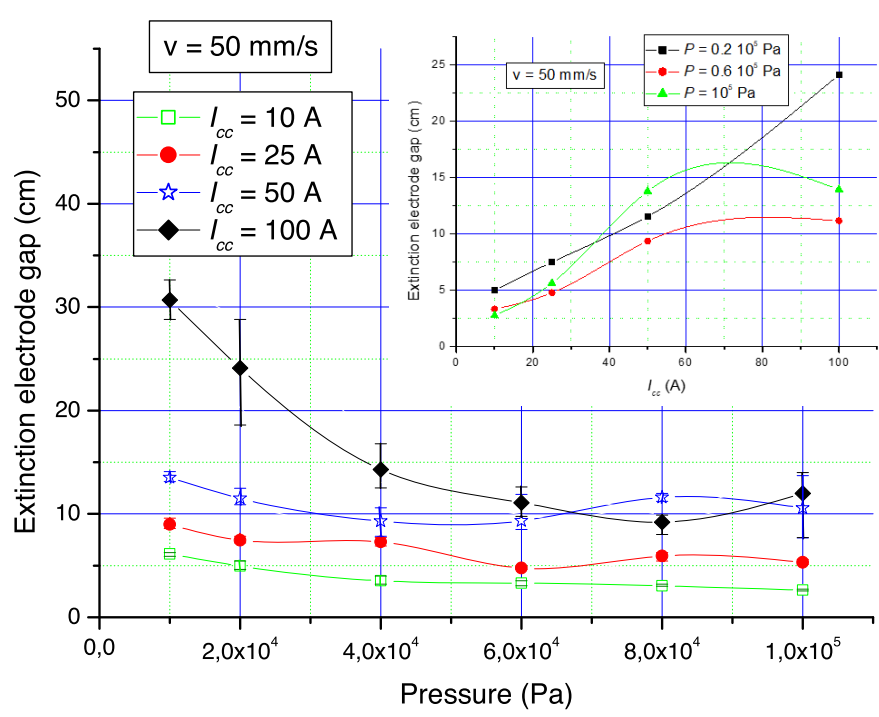

Fig. 10. Electrode gap at the electric arc extinction versus pressure for various $I_{\mathrm{CC}}$ values: $10,25,50,100 \mathrm{~A}$. The opening velocity is $50 \mathrm{~mm} / \mathrm{s}$.

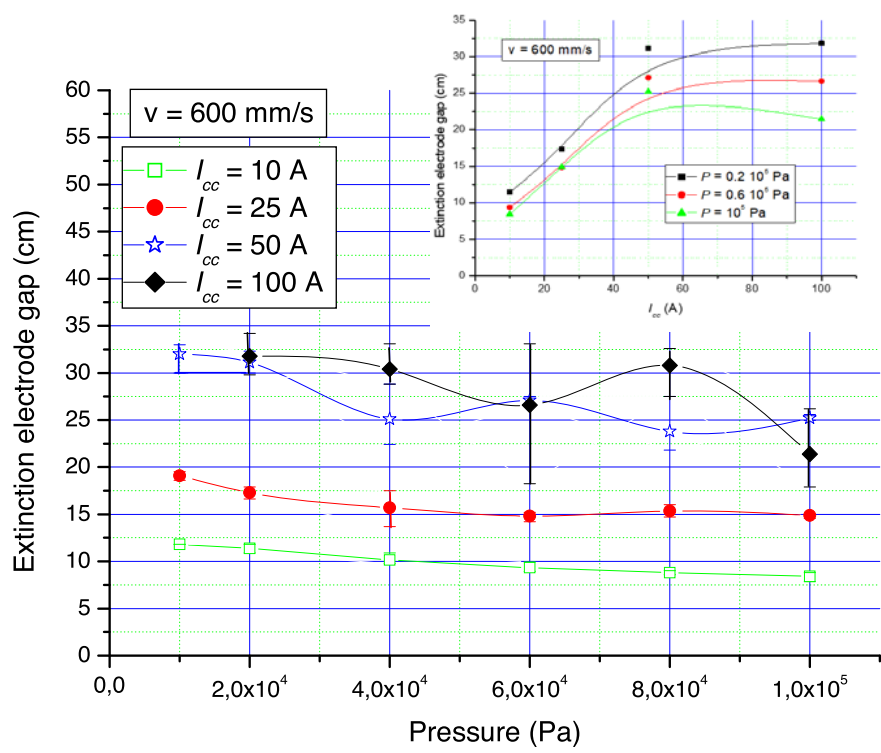

Fig. 11. Electrode gap at the electric arc extinction versus pressure for various $I_{\mathrm{CC}}$ values: $10,25,50,100 \mathrm{~A}$. The opening velocity is $600 \mathrm{~mm} / \mathrm{s}$.

In Figure 11, the experimental setup of $P=10^{4} \mathrm{~Pa}$, $I_{\mathrm{CC}}=100 \mathrm{~A}$ and $v=600 \mathrm{~mm} / \mathrm{s}$, leads to arcs for which the electrode gap is superior to the maximum electrode gap allowed by our vacuum chamber. As a result no measuring point appears in these experimental conditions.

In Figure 10, it can be underlined that for current values up to $50 \mathrm{~A}$, the extinction electrode gap is quite independent on pressure. The dependency on pressure is remarkable only for the highest current value considered in this work, that is to say $100 \mathrm{~A}$ and for low pressure, that is to say under $4 \times 10^{4} \mathrm{~Pa}$. 
On the top right of this figure, we have plotted the extinction electrode gap as a function of the current value $I_{\mathrm{CC}}$ for 3 values of pressure. It can be remarked that for current values under $50 \mathrm{~A}$, the relation between extinction electrode gap and the current $I_{\mathrm{CC}}$ is linear, for every pressure values considered. Contrarily, when considering current intensity values higher than $50 \mathrm{~A}$, two behaviors are observed: a linear relation for low pressure and a saturation for pressure from $600 \mathrm{hPa}$ to atmospheric pressure. The increase of separation speed promotes the saturation phenomenon, even at high current.

When the electrode separation velocity equals to $600 \mathrm{~mm} / \mathrm{s}$ as it is plotted in Figure 11, it can be observed that the extinction electrode gap is quite independent on pressure for every current value. When we look at the graph proposed in the top right of this figure, it is worth to note that a saturation phenomenon exists: the extinction electrode gap remains about constant for current values higher than $50 \mathrm{~A}$ and it is true for every pressure values.

It is important to underline that the separation velocity has an influence on the extinction electrode gap. Its increase from $50 \mathrm{~mm} / \mathrm{s}$ to $600 \mathrm{~mm} / \mathrm{s}$ causes $100 \%$ of increase at least on the extinction electrode gap of the arc.

Considering the opening velocities presented here and the values of the extinction electrode gap, it is interesting to note that the arc duration can reach several seconds: for example, at $v=50 \mathrm{~mm} / \mathrm{s}, I_{\mathrm{CC}}=100 \mathrm{~A}$ and $P=10^{4} \mathrm{~Pa}$, the duration is close to $6.3 \mathrm{~s}$.

\subsubsection{Observations and comments}

- It is necessary to recall that in the case of low separation velocity $(v=50 \mathrm{~mm} / \mathrm{s})$ the arc length may be very different from the electrode gap.

- As extinction electrode gap and opening speed are proportional, comments concerning arc extinction can be applied to arc duration.

- It is important to emphasize that the extinction electrode gap of the arc can easily exceed ten or twenty centimeters allowing then the electric arc to have interactions with its environment.

- The mean extinction length of the arc is all the more important as the separation velocity is important. Different explanations concerning the influence of the opening speed may be proposed. The duration of the arc for low speed is much longer than the duration for high speeds. This leaves as much time for the development of phenomena contributing to the deformation of the arc which can be of various origins: magneto hydrodynamics, deformation of the column under the effect of free thermal convection, which arises from the interaction between the cold gas and the hot plasma of the arc column [15]. In our case, the orientation of the electrodes (and so of the electric arc itself) is horizontal. It leads to a curvature of its column which could be different in a vertical orientation.

- Overall the extinction distance is a decreasing function of the pressure. High current values, low separation velocity of the electrodes and low pressure values are experimental conditions for which the pressure is the most influent.

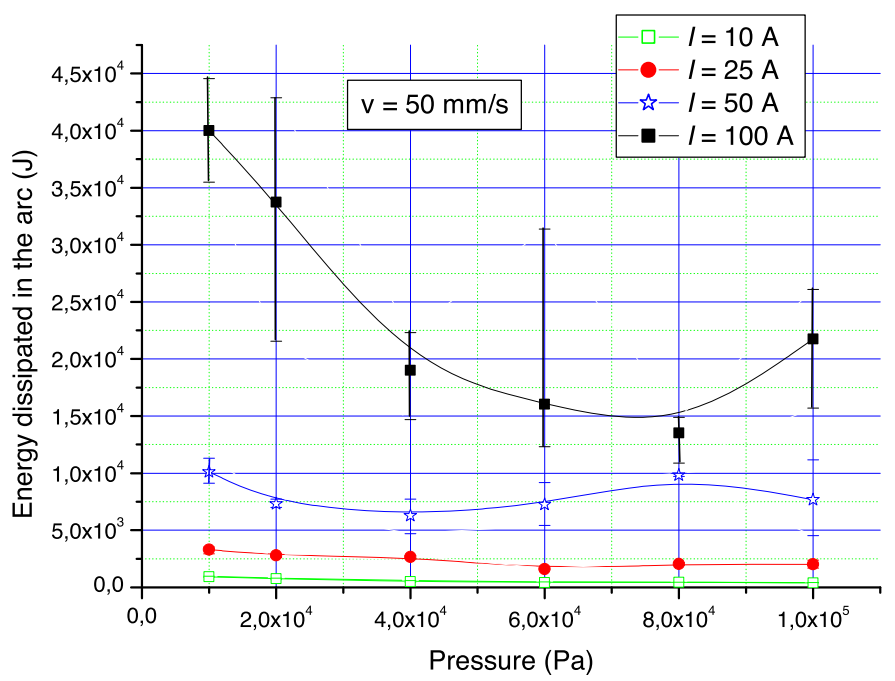

Fig. 12. Energy dissipated in the arc versus pressure for various $I_{\mathrm{CC}}$ values $(v=50 \mathrm{~mm} / \mathrm{s})$.

- It can be seen that when $I_{\mathrm{CC}}$ increases beyond a certain value, i.e. $50 \mathrm{~A}$, the extinction distance of the arc can be considered as constant. For other electrode materials several authors [14] observed that for $I_{\mathrm{CC}}$ smaller than $40 \mathrm{~A}$ the arc duration was an increasing function of $I_{\mathrm{CC}}$. Besides, the constancy of the extinction distance has already been observed at atmospheric pressure for supply voltages of the order of $40 \mathrm{~V} \mathrm{DC} \mathrm{[16]} \mathrm{and} \mathrm{was} \mathrm{attributed}$ to the appearance of instabilities of electromagnetic origin which destabilize the arc.

- At high current value, the error bars are large because of instabilities which induce less reproducible measurement than at low current.

\subsection{Arc energy dissipated in the arc versus pressure}

In Figures 12 and 13 the energy dissipated in the arc has been plotted versus the pressure for various arc current intensities and for respectively two separation velocities $(v=50 \mathrm{~mm} / \mathrm{s}$ and $600 \mathrm{~mm} / \mathrm{s})$. On one hand, for current values under $50 \mathrm{~A}$ (included), we notice that the variation of pressure does not carry a great variation on the energy dissipated in the electric arc. On the other hand, for a current of $100 \mathrm{~A}$, the impact of the pressure variation is considerably high, especially for low pressures.

For both opening velocities considered and for current values under $50 \mathrm{~A}$ (included), it can be remarked that the pressure does not have a drastic influence on the energy dissipated in the arc. Moreover, when the opening velocity is low, the arc lives longer so that it can carry higher energy. An increase in the arc duration results in a more convective arc column. This convection phenomena governs the arc column length and it can be understood that more energy is required to maintain a longer arc.

The energy in the arc for a separation speed of $50 \mathrm{~mm} / \mathrm{s}$ is significantly higher than that of $600 \mathrm{~mm} / \mathrm{s}$, this can be explained by the fact that the arc duration is greater for the separation speed at $50 \mathrm{~mm} / \mathrm{s}$. Another factor that can increase the energy dissipated in the arc in the case of the 


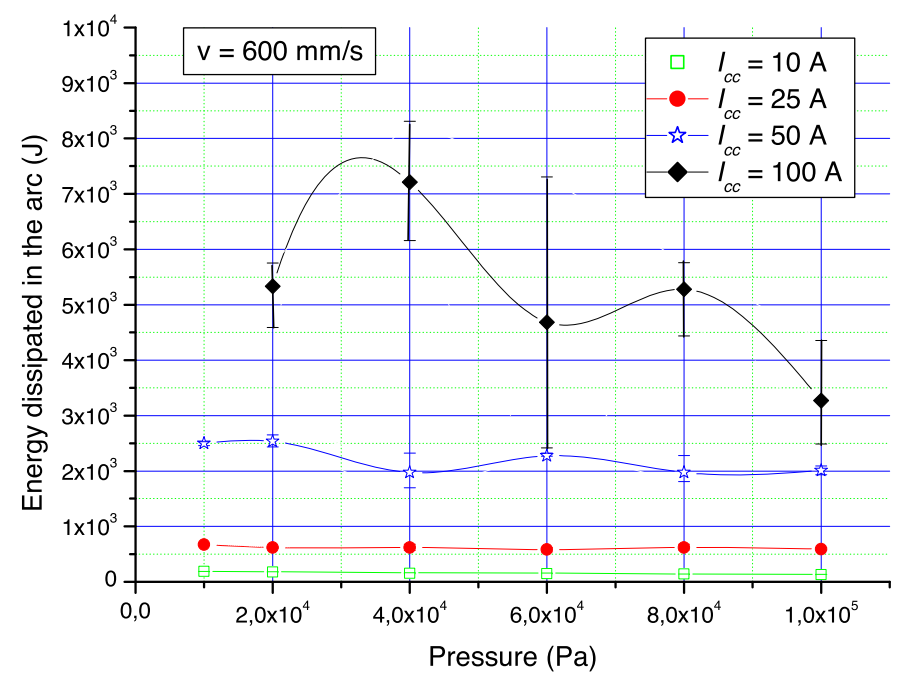

Fig. 13. Energy dissipated in the arc versus pressure for various $I_{\mathrm{CC}}$ values $(10,25,50,100 \mathrm{~A})$. The opening velocity is $600 \mathrm{~mm} / \mathrm{s}$.

separation speed of $50 \mathrm{~mm} / \mathrm{s}$ is the presence of arc instabilities. These instabilities are characterized by an extension of the arc column comparatively to the electrode gap. The direct consequence of this extension is a larger voltage drop in the arc column which generates more energy dissipation in the arc column. On the contrary, in the case of a separation speed of $600 \mathrm{~mm} / \mathrm{s}$, the length of the arc is taken equal to the inter electrode distance.

\subsubsection{Observations and comments}

- The energy dissipated in the arc may reach very important value ( $10 \mathrm{~kJ}$ or more).

- As already observed for lower supply voltage (40 V DC) [1], the energy does not depend strongly on pressure values for small current intensities.

- Comparing the present results to those presented in [1], the influence of the supply voltage must be mentioned: in [1], for a supply voltage of $36 / 42 \mathrm{~V} \mathrm{DC}$ and $I_{\mathrm{CC}}=70 \mathrm{~A}$ (and $v=300 \mathrm{~mm} / \mathrm{s}$ ), the mean energy dissipated in the arc varies with pressure between 6 and $12 \mathrm{~J}$. In the present case, for $I_{\mathrm{CC}}=50 \mathrm{~A}$ (and $v=50 \mathrm{~mm} / \mathrm{s}$ ), it varies in the range [5-10] kJ, practically 1000 times greater. Similarly, in [1] for $I_{\mathrm{CC}}=300 \mathrm{~A}$ the mean energy varies in the range $[60-120] \mathrm{kJ}$. In this work for $I_{\mathrm{CC}}=100 \mathrm{~A}$ it varies in the range [13-40] kJ. From these data, it can be remarked that the influence of voltage increase does not lead to a linear increase of the energy dissipated by the arc. When the order of magnitude of the voltage supply is multiplied by a factor 10 , the order of magnitude of the energy dissipated by the arc is multiplied by a factor 1000 .

- Energy, extinction electrode gap and arc duration change in a similar way with pressure.

In Figure 14, we have plotted the mean linear power density dissipated in the arc for the maximal separation velocity, i.e. $v=600 \mathrm{~mm} / \mathrm{s}$. It shows then clearly the influence of the pressure on this parameter: the higher the pressure, the more the arc is energetic.

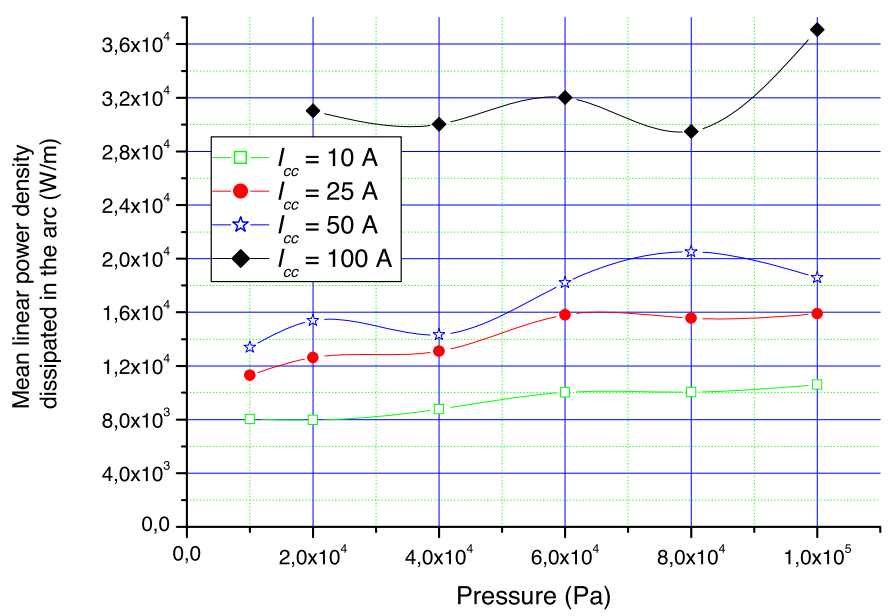

Fig. 14. Mean linear power density dissipated in the arc versus pressure for various $I_{\mathrm{CC}}$ values $(10,25,50,100 \mathrm{~A})$. The opening velocity is $600 \mathrm{~mm} / \mathrm{s}$.

\section{Conclusion}

In this work, we have studied the influence of pressure, of current intensity value and of the separation velocity on the length of extinction of an electric arc. The main idea was to underline the behavior of electric arcs with pressure variations, for several current intensity values and by considering typical speed values of disconnection (falling cable for example). Estimations of the energy dissipated per arc and of the mean linear power density per arc have been proposed. The experimental conditions for which the arc can extend spatially the more are: low pressure, maximum disconnection speed, maximum current intensity. As a conclusion concerning more electrical aircrafts, the increasing voltages will increase the length, duration and energy of electrical arcs. Consequently to these facts, the potential damage onboard will grow. The spatial extension of electric arcs for reduced pressures will make circuit breaking difficult. New breakers will have to be built. More than ever, it will be needy to break electric arc as soon as possible, which gives all its sense to new detection techniques that will have to be developed.

\section{Author contribution statement}

Please note that you are required to include a statement which details the nature of the contribution of each author.

\section{References}

1. R. Landfried et al., Eur. Phys. J. Appl. Phys. 67, 20802 (2014)

2. C.P. McCabe et al., A study of over-current protection, electrical arcing, and fire protection, in 51st AIAA / ASME/ ASCE/AHS/ASC Structures, Structural Dynamics, and 
Materials Conference 18th, 12-15 April 2010, Orlando, Florida

3. V.E. Phillips et al., Trans. AIEE 63, 944 (1944)

4. J.S. Quill et al., Trans. AIEE 63, 883 (1944)

5. T.R. Stuelnagel, Trans. AIEE 69, 1289 (1950)

6. B.S. Beal et al., Electr. Eng. 71, 776 (1952)

7. T. Klonowski et al., Characterization of HVDC contactors in depressurized environment, More Electrical Aircraft, Toulouse 5-6 février 2019

8. J. Swingler et al., The evolution of contact erosion during an opening operation at $42 \mathrm{~V}$, in Proceedings of 51th IEEE Holm Conference on Electrical Contacts, 2005

9. T.J. Schoepf, Electrical contacts in the automotive $42 \mathrm{~V}$ DC PowerNet, in Proceedings of the 21th International Conference on Electrical Contact, 2002, pp. 43-55

10. L. Doublet et al., Electrical arc phenomena and its interaction on contact material at $42 \mathrm{~V}$ DC for automotive applications, in Proceedings of the 50th IEEE Holm Conference on Electrical Contact, 2004, pp. 8-14
11. P. Colombo et al., Arcing study in high current electromechanical switching for future 14-4 2V Dual Voltage Systems, SAE Technical Paper 2002-01-0520, 2002

12. P. Teste et al., Eur. Phys. J. Appl. Phys. 41, 251 (2008)

13. R. Boukadoum et al., Role of the pressure in the DC electric arc characteristics application: case of the more electrical aircraft, in Proceedings of 28th International Conference on Electrical Contact, 2016, pp. 153-158

14. J.W. McBride et al., The influence of contact opening velocity on arc characteristics, in Proceedings the 16th International Conference on Electrical Contact, 1992, pp. 395-400

15. A.M. Howatson et al., J. Phys. D: Appl. Phys. 9, 1101 (1976)

16. T. Klonowski et al., IEEE Trans. Vehic. Technol. 56, 2017 (2007)

Open Access This article is distributed under the terms of the Creative Commons Attribution License https://creativecom mons.org/licenses/by/4.0 which permits unrestricted use, distribution, and reproduction in any medium, provided the original author(s) and source are credited.

Cite this article as: Romaric Landfried, Mohamed Boukhlifa, Thierry Leblanc, Philippe Teste, Jonathan Andrea, Stability, spatial extension extinction of an electric arc in aeronautical conditions of pressure under 540 V DC, Eur. Phys. J. Appl. Phys. 87, 30901 (2019) 